\title{
Three-Dimensional Electrostatic Field at an Electron Nano-Emitter Determined by Differential Phase Contrast in Scanning Transmission Electron Microscopy
}

\author{
Mingjian $\mathrm{Wu}^{1 *}$, Alexander Tafel $^{2}$ Peter Hommelhoff $^{2}$ and Erdmann Spiecker ${ }^{*}$ \\ 1. Institute of Micro- and Nanostructure Reseaerch, Center for Nanoanalysis and Electron Microscopy \\ (CENEM), University of Erlangen-Nuremberg, Erlangen, Germany. \\ 2. Institute of Laser Physics, University of Erlangen-Nuremberg, Erlangen, Germany. \\ * Corresponding authors: Mingjian.wu@fau.de; Erdmann.Spiecker@fau.de
}

Revealing and quantifying the 3-dimensional (3D) electrostatic field of field emission nano-emitters is key to answer the fundamental question of how the field interacts with the sharp tips, which has not yet been adequately addressed experimentally despite its long history. Here, we determine the 3D electrostatic field in situ in the transmission electron microscope (TEM) at an electron nano-emitter.

A sharp tungsten tip with an apex radius of about $20 \mathrm{~nm}$ is inserted at the movable side in a Nanofactory STM-TEM holder as cathode and a gold rod as grounded anode, between which the distances can be well controlled. Differential phase contrast (DPC) in scanning TEM (STEM) mode, using a quadrant segmented annular detector, has been applied to map the (projected) electron beam deflection due to the field around the tip. With careful calibration and optimizing the imaging conditions, the (projected) beam deflection could be measured to high precision (order of $10^{-6} \mathrm{rad}$, condition dependent) and wide linear signal range (up to few $10^{-3} \mathrm{rad}$ ). After every experimental data acquisition, a vacuum area scan is recorded and used as reference to correct the non-perfect scan pivot point when scanning over large field-of-view. Both are essential to reveal the locally strong and extended field (where field-of-view is needed) as in the case of field emission. Furthermore, we derived a method based on the inverse Abel transform to quantitatively reconstruct an axial slice of the 3D electrostatic field from a single projection measurement assuming axial symmetry of the nano-emitter (Fig. 1). Under condition of a bias voltage of $140 \mathrm{~V}$ with respect to the grounded counter electrode located about $650 \mathrm{~nm}$ from the apex The highest field strength of $2.92 \mathrm{~V} / \mathrm{nm}$ is measured at the nano-emitter apex, resulting in an emission current of more than $2 \mathrm{uA}$. The experimental results are compared with simulations based on a finite element numerical Maxwell equation solver. Quantitative agreement between experiment and simulation has been achieved (Fig. 2).

In contrast to previous techniques based on electron holography, problems with reference waves distortion are circumvented in DPC enabling straightforward interpretation of the data. DPC also allows for a balance of field of view with resolution as well as an easily calibrated signal. We believe the technique will also be applicable to other systems such as studying fields around tips of general shape, provided that tilt series data acquisition would be feasible.

References:

[1] M. Wu, A. Tafel, P. Hommelhoff and E. Spiecker, Applied Physics Letters 114 (2019) 013101.

[2] M.W. and E.S. acknowledge financial support by DFG via research training group GRK 1896 "Insitu Microscopy with Electrons, X-rays and Scanning Probes" and usage of instrumentation acquired within the DFG Cluster of Excellence EXC 315 "Engineering of Advanced Materials". A.T. and P.H. acknowledge funding by the ERC grant "Near Field Atto." 

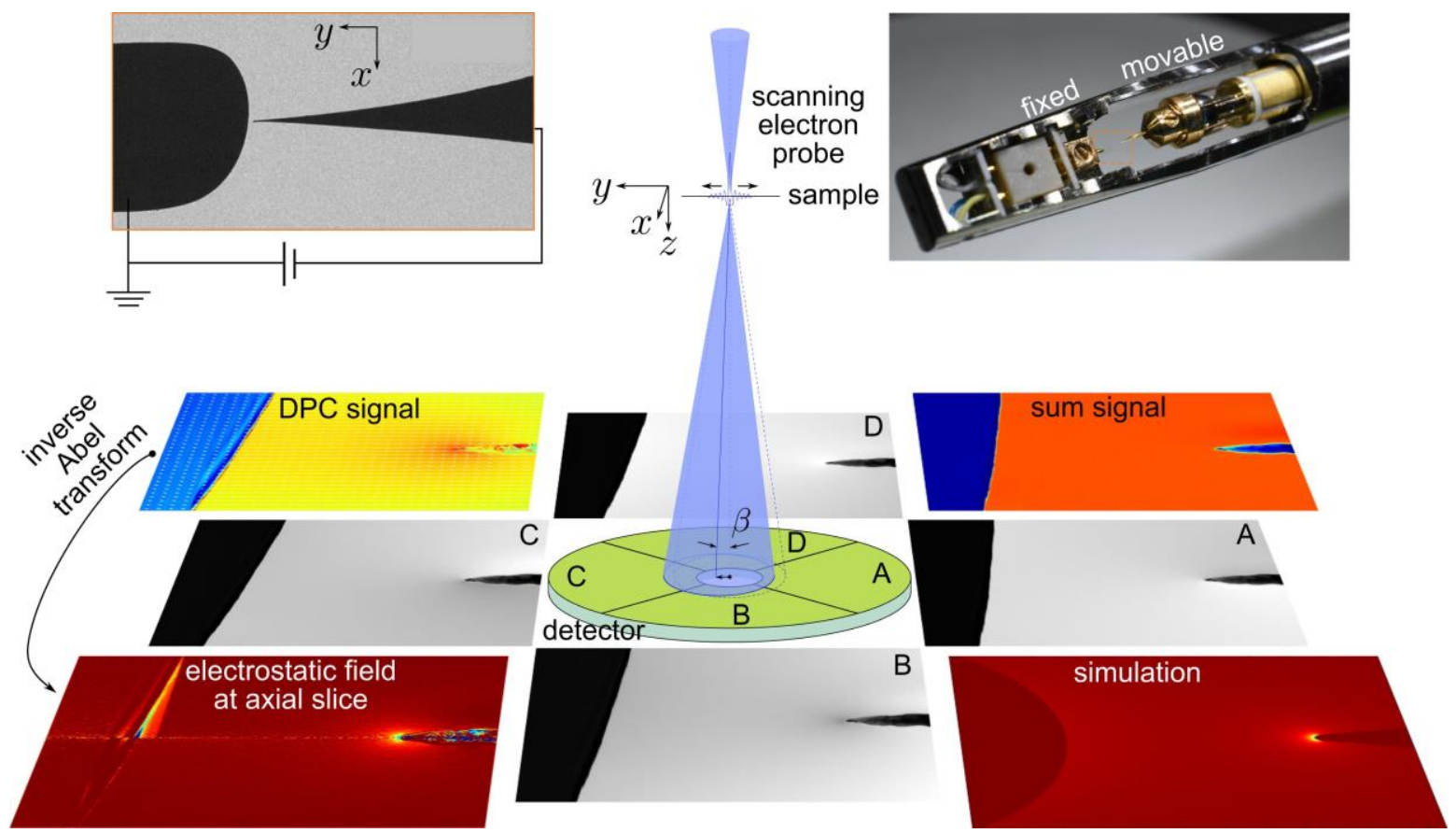

Figure 1. Schematic illustration of the experimental setup. The DPC signal, as a projection measurement of electron beam deflection while it pass through the electrostatic field, is calculated and quantified from the four images as obtained by the quadrant detector segments. Assuming a cylindrical symmetry of the nano-emitter, the 3D electrostatic field is reconstructed based on inverse Abel transformation. The results show excellent agreement to simulation.

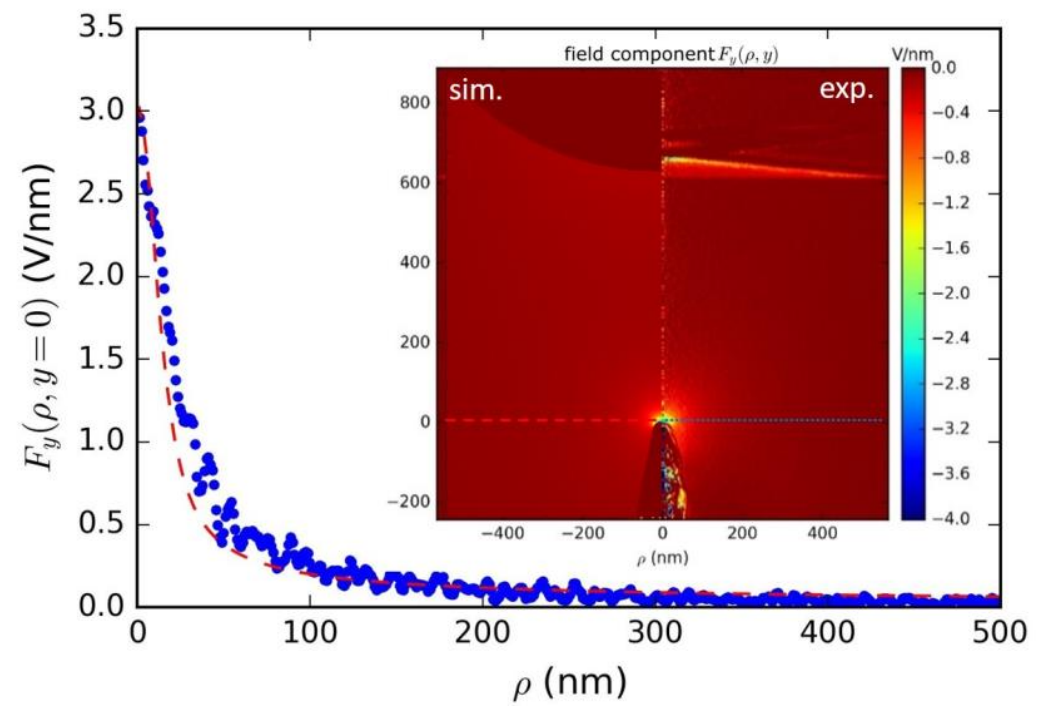

Figure 2. Comparison of the electrostatic field experimentally determined and from simulation. The plot show the field component normal to the emission tip surface as a function of the distance from tip to radial direction (the red and blue line directions marked in the inset). 